\title{
Tessaracoccus bendigoensis gen. nov., sp. nov., a Gram-positive coccus occurring in regular packages or tetrads, isolated from activated sludge biomass
}

1 Biotechnology Research Centre, La Trobe University, Bendigo, VIC 3550, Australia

2 Faculty of Science, School of Biomolecular and Biomedical Sciences, Griffith University, Nathan, Brisbane, QLD 4111, Australia

3 Deutsche Sammlung von Mikroorganismen und Zellkulturen $\mathrm{GmbH}$, Aussenstelle Jena, Beutenbergstrasse 11, D-07745 Jena, Germany

4 CRC Freshwater Ecology, Murray-Darling Freshwater Research Centre, PO Box 921, Albury, NSW 2640, Australia

\author{
A. M. Maszenan, ${ }^{1}$ R. J. Seviour, ${ }^{1}$ B. K. C. Patel, ${ }^{2}$ P. Schumann ${ }^{3}$ and G. N. \\ Rees $^{4}$
}

Author for correspondence: R. J. Seviour. Tel: +61 354447459 . Fax: +61 354447476 e-mail: r.seviour@latrobe.edu.au

\begin{abstract}
An isolate of a Gram-positive bacterium, designated strain Ben 106', was obtained in pure culture by micromanipulation of a biomass sample obtained from a laboratory-scale sequencing batch reactor. This isolate grew axenically as cocci or clusters of cocci arranged in regular tetrads and was morphologically similar to the dominant organism observed in the biomass. This morphology resembled that of some Gram-positive and -negative bacteria and the so-called ' G-bacteria' commonly seen in activated sludge samples. Strain Ben ${ }^{106}{ }^{\top}$ is a non-motile, facultative anaerobe. It is oxidase-negative, catalase-positive and is capable of reducing nitrate. This organism can grow between 20 and $37{ }^{\circ} \mathrm{C}$, with an optimum temperature of $25^{\circ} \mathrm{C}$. The $\mathrm{pH}$ range for growth is between 6.0 and 9.0, with an optimum pH of 7.5. The isolate stained positively for intracellular polyphosphate granules. The diagnostic diamino acid of the peptidoglycan is LL-diaminopimelic acid (LL-A $\mathbf{p m}$ ) with a glycine moiety at position 1 of the peptide subunit, which characterizes the presence of a rare peptidoglycan (type A3- $\left.\gamma^{\prime}\right)$. Two menaquinones, MK-9 $\left(\mathrm{H}_{4}\right)$ and MK$7\left(\mathrm{H}_{4}\right)$, are present and the main cellular fatty acid is 12-methyltetradecanoic acid. The G+C content is $74 \mathrm{~mol} \%$. From phenotypic characteristics and $16 \mathrm{~S}$ rDNA sequence analysis, the isolate differed sufficiently from its closest phylogenetic relatives, namely Propionibacterium propionicum, Propioniferax innocua, Friedmanniella antarctica, Luteococcus japonicus and Microlunatus phosphovorus in the A1 subdivision of the Gram-positive bacteria (i.e. Firmicutes with a high $\mathrm{G}+\mathrm{C}$ content), suborder Propionibacterineae, to be placed in a new genus, Tessaracoccus, as Tessaracoccus bendigoensis gen. nov., sp. nov. The type strain is Ben 106' (= ACM 5119').
\end{abstract}

Keywords: Tessaracoccus bendigoensis, activated sludge, Gram-positive coccus, Propionibacteriaceae, G-bacteria

\section{INTRODUCTION}

Many early studies on bacterial populations in activated sludge systems have reported the isolation and identification of members of the genus Micrococcus (Painter, 1983; Wanner, 1994), a Gram-positive coccus

\footnotetext{
Abbreviations: LL- $\mathrm{A}_{2} \mathrm{pm}$, LL-diaminopimelic acid; PHA, polyhydroxyalkanoate; PolyP, polyphosphate; SBR, sequencing batch reactor.

The GenBank accession number for the 165 rRNA sequence of Tessaracoccus bendigoensis strain Ben $106^{\top}$ is AF038504.
}

often arranged distinctively in tetrads. Gram-negative cocci, generically described as 'G-bacteria' (Carrucci et al., 1994; Cech \& Hartman, 1993), have also been seen frequently in large numbers in biomass samples from plants around the world. Some of these Gbacteria have been successfully cultured (Cech \& Hartman, 1993; Maszenan et al., 1997) and four isolates have recently been characterized and described as belonging to a new genus Amaricoccus (Maszenan et al., 1997).

Cocci other than Amaricoccus spp. and Micrococcus spp. occur in activated sludge systems and the identity 
of some of these cocci is now known. For example, a Gram-positive coccus which can accumulate polyphosphate was identified as the new bacterium Microlunatus phosphovorus in Japan (Nakamura et al., 1991, 1995a, b; Ubukata \& Takii, 1994; Ubukata, 1994) and Microsphaera multipartita was also obtained from activated sludge biomass from a plant treating carbohydrate-rich wastewater (Takii, 1977a, b; Yoshimi et al., 1996). Neither of these organisms have so far been reported in plants in other parts of the world. Kataoka et al. (1996) successfully isolated a Micrococcus-like bacterium by culturing activated sludge biomass under starvation conditions prior to isolation, but did not identify the isolate (Kataoka et al., 1996). Grampositive tetrad cocci are also commonly encountered in biomass from sequencing batch reactors designed for phosphorus removal, but the identities of these organisms remain unknown (Liu et al., 1996; Matsuo, 1994; Matsuzawa \& Mino, 1991; Randall, 1994).

The activated sludge system is expected to harbour a wide spectrum of previously undescribed bacteria, so the Gram-positive cocci present may be very diverse and represent novel taxonomic and phylogenetic groups. Until the true diversity of bacteria in activated sludge is better understood and their physiology and ecology are studied, our ability to optimize and control these treatment systems will be limited. As part of our ongoing project to investigate these groups of bacteria, we used micromanipulation (Skerman, 1968) to isolate an organism with cocci in a distinctive tetrad arrangement. This organism was designated strain Ben $106^{\mathrm{T}}\left(\mathrm{ACM} 5119^{\mathrm{T}}\right)$. The phenotypic, chemotaxonomic and phylogenetic properties indicate that strain Ben $106^{\mathrm{T}}$ cannot be assigned to any known genus and should be placed in a new genus, Tessaracoccus, as Tessaracoccus bendigoensis gen. nov., sp. nov.

\section{METHODS}

Isolation and maintenance of strain Ben $106^{\top}$. Strain Ben $106^{\mathrm{T}}$ (ACM 5119 ) was isolated using a Skerman micromanipulator (Skerman, 1968) from activated sludge biomass from a laboratory-scale sequencing batch reactor (SBR) seeded with activated sludge obtained from the Biological Nutrient Removal (BNR) plant in Bendigo, Australia. The SBR (8 1 total vol.) was operated under an anaerobic/aerobic cycle regime consisting of $90 \mathrm{~min}$ anaerobic, $240 \mathrm{~min}$ aerobic periods and a 30 min settle with a subsequent decant period. Four litres of effluent were decanted at the end of each cycle and the mean sludge age was $8 \mathrm{~d}$. The synthetic feed used consisted of $\left(\mathrm{l}^{-1}\right)$ sodium acetate $(100 \mathrm{mg})$, glucose $(400 \mathrm{mg})$, $\mathrm{KH}_{2} \mathrm{PO}_{4}(57 \mathrm{mg}),\left(\mathrm{NH}_{4}\right)_{2} \mathrm{SO}_{4}(100 \mathrm{mg}), \mathrm{NaCl}(100 \mathrm{mg})$, $\mathrm{CaCl}_{2}(50 \mathrm{mg}), \mathrm{NaHCO}_{3}(200 \mathrm{mg})$ and $\mathrm{MgSO}_{4}(80 \mathrm{mg})$. The SBR was maintained at $20^{\circ} \mathrm{C}$. Pure cultures were obtained by streaking out colonies arising from micromanipulated cells several times onto fresh GS medium (Williams \& Unz, 1985 ) and incubating plates at $25^{\circ} \mathrm{C}$. Purity was confirmed by microscopic examination of cells from single colonies. Strain Ben $106^{\mathrm{T}}$ was stored on GS medium in $20 \%$ glycerol at $-80^{\circ} \mathrm{C}$

Physiological and biochemical characterization of strain Ben $106^{\mathrm{T}}$. Substrate utilization patterns for strain Ben $106^{\mathrm{T}}$ were obtained with both GP and GN Biolog systems (Special Diagnostic) as described previously (Maszenan et al., 1997). Enzyme profiles were determined using API ZYM strips (bioMérieux) following the manufacturer's instructions. All biochemical properties were obtained with the Microbact 24E system (Oxoid). Catalase, oxidase, motility tests and growth response to $\mathrm{pH}$ and temperature were performed as described by Maszenan et al. (1997). Detection of both polyphosphate (PolyP) and polyhydroxyalkanoate (PHA) granules were performed according to the method of Rees et al. (1992). Cultures for PolyP and PHA examination were grown on basal GS medium with glucose, acetate or propionate, all at $0.15 \mathrm{~g} \mathrm{l}^{-1}$, as carbon sources, at their optimal growth temperature. The possible presence of intracellular PolyP and PHA granules was determined using an Olympus fluorescent microscope (model BH-RFL) fitted with an L-410 exciter filter, L-420 barrier filter and G 580 chromatic beam splitter. The response of the organism to $\mathrm{O}_{2}$ was determined by stabbing the culture into a GS agar deep. Whether its metabolism was oxidative or fermentative $(\mathrm{O} / \mathrm{F})$ was determined with Hugh and Leifson $(\mathrm{O} / \mathrm{F})$ medium (Oxoid).

Chemotaxonomic characterization. The biomass used for chemotaxonomic characterization of Ben $106^{\mathrm{T}}$ was obtained by scraping cells from the surfaces of R-agar plates (Schumann et al., 1997) incubated at $22{ }^{\circ} \mathrm{C}$ for $2-3$ weeks. Cell wall and lipid components were analysed as described by Schumann et al. (1997). Amino acids and peptides in cell wall hydrolysates were analysed by two-dimensional TLC (Schleifer \& Kandler, 1972). Cellular fatty acid methyl esters were separated by GC using a non-polar (type OV-1) capillary column $(25 \mathrm{~m} \times 0.25 \mathrm{~mm}$ i.d.). Polar lipids were separated by two-dimensional TLC on silica gel and identified by spraying with specific reagents and menaquinones were analysed by reversed-phase HPLC (Schumann et al., 1997).

Preparation of specimens for scanning electron microscopy. Samples were prepared for examination by scanning electron microscopy according to the method of Maszenan et al. (1997).

16S rDNA sequence and phylogenetic analysis. 16S rDNA amplification and sequencing were carried out as already described by Maszenan et al. (1997). Sequences were aligned with genera of the domain Bacteria and Archaea using the methods of Patel et al. (1995). Pairwise evolutionary distances were computed according to the method of Jukes \& Cantor (1969). Dendrograms were constructed from evolutionary distances with the neighbour-joining method. Tree topology was examined with 100 bootstrapped data sets. The computer programs used form part of the PHYLIP program version 3.51c (Felsenstein, 1993).

$\mathbf{G}+\mathbf{C}$ content. The DNA base composition was calculated from the melting temperature determined by the thermal denaturation method of Owen \& Lapage, (1976). Cell DNA was isolated using the method of Porteous et al. (1994), modified by disrupting the cells with a cell homogenizer (Braun) and incubating them with $50 \mathrm{mg}$ lysozyme $\mathrm{ml}^{-1}$ (Sigma) and $100 \mathrm{U}$ achromopeptidase $\mathrm{ml}^{-1}$ (Sigma) for $60 \mathrm{~min}$ at $37^{\circ} \mathrm{C}$ before DNA extraction (Porteous et al., 1994). This method was used to overcome difficulties associated with the production of large amounts of extracellular polysaccharide material by this organism. Disrupted cells were incubated with $5 \mathrm{M}$ guanidinium thiocyanate (Promega) for $60 \mathrm{~min}$ and the DNA spooled onto a sterile glass pipette. 


\section{RESULTS}

\section{Culture and morphological characteristics}

The isolation of strain Ben $106^{\mathrm{T}}$ was difficult. A wide range of media routinely used for isolation of bacteria from activated sludge was tested. Only GS medium was successful in supporting the growth of micromanipulated cells. The organism grew as beige colonies, visible after $2-3 \mathrm{~d}$ incubation at $25^{\circ} \mathrm{C}$ and shared the distinctive coccal morphology of cells in tetrads as seen in large numbers in the SBR biomass (Fig. 1). The diameter of the individual coccoid cells ranged from 0.5 to $1 \cdot 1 \mu \mathrm{m}$. Cells could accumulate intracellular PolyP granules aerobically with all three carbon sources examined (Fig. 1d) but PHA granules were not observed.

\section{Physiological and biochemical characteristics}

Results obtained with the Biolog GN and GP systems and Microbact 24E system showed that strain Ben $106^{\mathrm{T}}$ could utilize L-arabinose, D-ribose, D-fructose, D- galactose, gentiobiose, $\alpha$-D-glucose, $\alpha$-D-lactose, lactulose, maltose, D-mannose, D-melibiose, sucrose, turanose, D-raffinose, D-xylose, stachyose, palatinose, maltotriose, methyl $\alpha$-D-glucoside, methyl $\alpha$-D-galactoside, methyl $\beta$-D-galactoside, $\alpha$-cyclodextrin, $\beta$-cyclodextrin, dextrin, amygladin, arbutin, glycogen, D-mannitol, methylpyruvate, glucose 1-phosphate, glucose 6-phosphate, DL-glycerol phosphate, UMP, glycerol, uridine and salicin. Strain Ben $106^{\mathrm{T}}$ could not metabolize the following sugars and sugar derivatives: cellobiose, L-fucose, D-psicose, L-rhamnose, D-trehalose, D-melezitose, 3-methylglucose, methyl $\beta$-D-glucoside, mannan, adonitol, D-arabitol, i-erythritol, $m$-inositol, D-sorbitol, xylitol, monomethylsuccinate, D-galacturonic acid lactone, 2-aminoethanol, 2,3-butanediol, inulin, fructose 6-phosphate, methylsuccinate, adenosine, 2'-deoxyadenosine, AMP, TMP, D-tagatose, methyl $\alpha$-D-mannoside and sedoheptulosan.

The following acids were metabolized by Ben $106^{\mathrm{T}}$ : acetic acid, D-gluconic acid, $\alpha$-hydroxybutyric acid, DL-lactic acid, L-lactic acid and pyruvic acid. None of
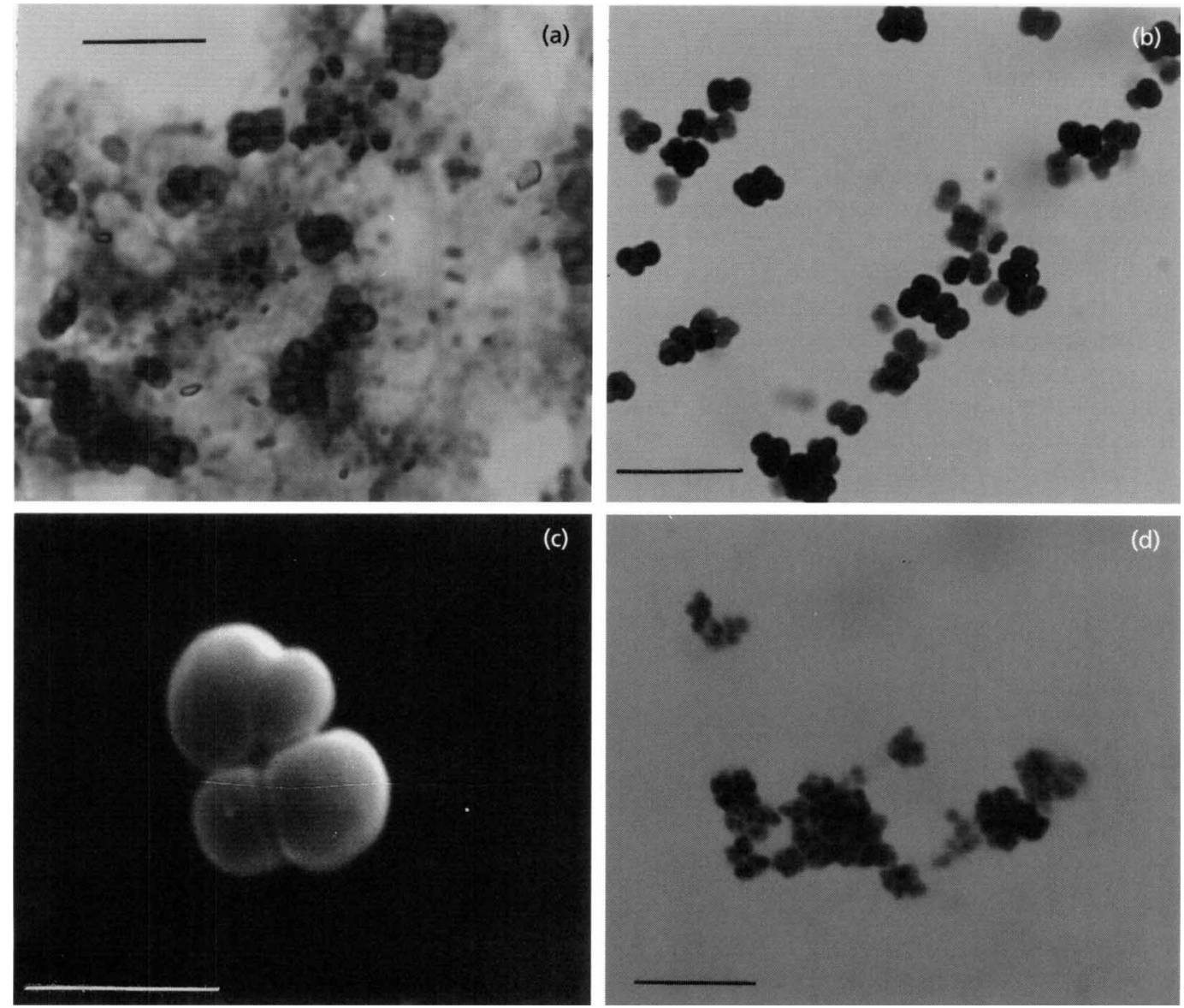

Fig. 1. Morphology of Gram-positive cocci obtained from an activated sludge sample. (a) Cells arranged in tetrads in activated sludge biomass from a laboratory-scale SBR, Bendigo, Australia. Bar, $10 \mu \mathrm{m}$. (b) Light micrograph of strain Ben $106^{\top}$ following Gram staining. Bar, $10 \mu \mathrm{m}$. (c) Scanning electron micrograph of strain Ben $106^{\top}$ showing the characteristic tetrad morphology. Bar, $2 \mu \mathrm{m}$. (d) Light micrograph of strain Ben $106^{\mathrm{T}}$ stained with the polyphosphate stain of Rees et al. (1992). Bar, $10 \mu \mathrm{m}$. 


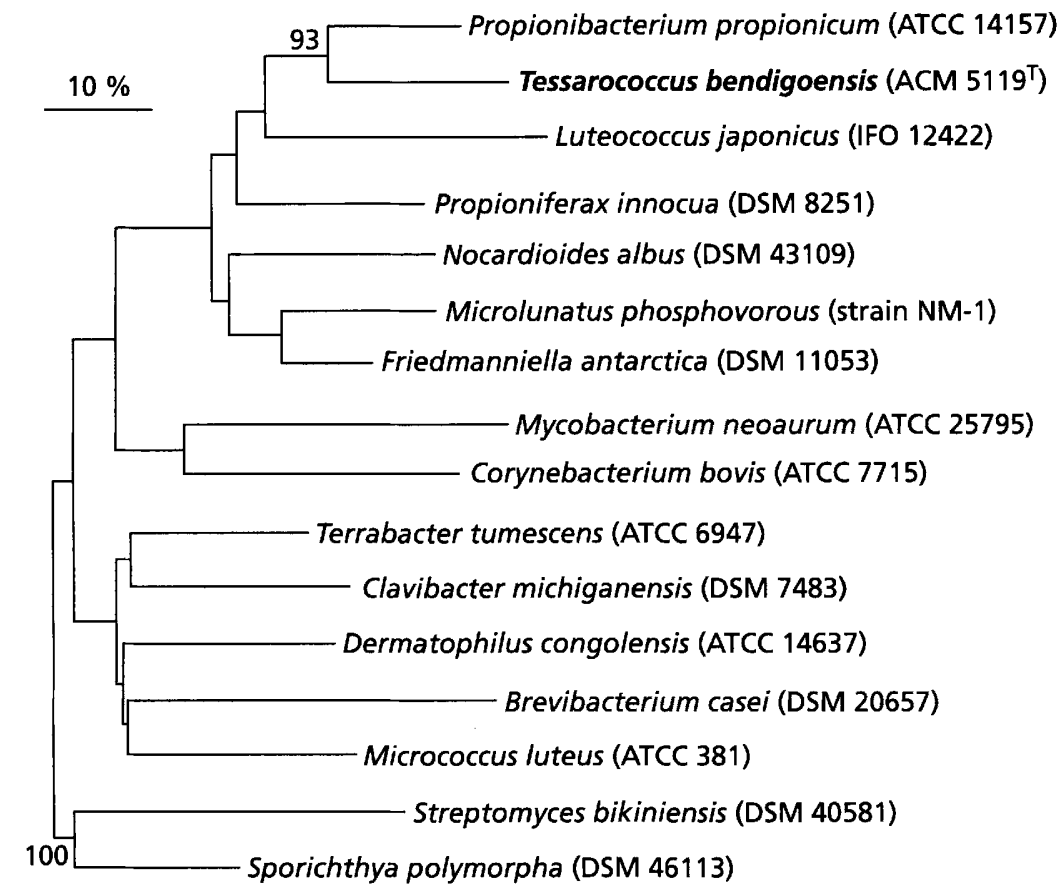

Fig. 2. Phylogenetic dendrogram based on $16 \mathrm{~S}$ rRNA sequence data, indicating the position of Tessaracoccus bendigoensis (strain Ben $106^{\mathrm{T}}$ ) within the radiation of representatives of the high-G + C-containing Gram-positive bacteria. All the sequences used in the analysis were obtained from the Ribosomal Database Project, version 5.0 (Maidak et al., 1996). Bootstrap values, expressed as a percentage of 100 replications are shown at the branching points. Only values above $90 \%$ were considered significant and therefore reported. Scale bar indicates 10 substitutions per $100 \mathrm{nt}$.

the following acids, amino acids and their derivatives were metabolized by strain Ben $106^{\mathrm{T}}$ : cis-aconitic acid, citric acid, formic acid, D-galacturonic acid lactone, Dgalacturonic acid, D-glucosaminic acid, D-glucuronic acid, $\beta$-hydroxybutyric acid, $\gamma$-hydroxybutyric acid, $p$ hydroxyphenylacetic acid, itaconic acid, $\alpha$-ketobutyric acid, $\alpha$-ketoglutaric acid, $\alpha$-ketovaleric acid, malonic acid, propionic acid, quinic acid, D-saccharic acid, sebacic acid, succinic acid, bromosuccinic acid, succinamic acid, L-aspartic acid, L-glutamic acid, glycyl-Laspartic acid, glycyl-L-glutamic acid, L-pyroglutamic acid, $\gamma$-aminobutyric acid, uroconic acid, D-lactic acid methyl ester, D-malic acid, L-malic acid and $\mathrm{N}$-acetylL-glutamic acid.

$\mathrm{N}$-Acetyl-D-glucosamine was the only amine derivative metabolized by strain Ben $106^{\mathrm{T}}$, which was not able to metabolize the following amino acids, amines and their derivatives: $\mathrm{D}$-alanine, $\mathrm{L}$-alanine, $\mathrm{L}$-alanylglycine, L-asparagine, L-histidine, L-leucine, L-ornithine, Lphenylalanine, L-proline, D-serine, L-serine, L-threonine, DL-carnitine, inosine, thymidine, phenylethylamine, putrescine and hydroxy-L-proline. In addition, strain Ben $106^{\mathrm{T}}$ could not metabolize Tween 40 , Tween 80 , glucuronamide, alaninamide, lactamide, $N$-acetylD-galactosamine and $N$-acetylmannosamine.

The enzyme activities detected by both the API ZYM and Microbact 24E systems were alkaline phosphatase, esterase, esterase lipase, lipase, leucine arylamidase, valine arylamidase, cystine arylamidase, acid phosphatase, naphthol-AS-BI-phosphohydrolase, $\alpha$-galactosidase, $\beta$-galactosidase, $\alpha$-glucosidase, $\beta$-glucosidase, $N$-acetyl- $\beta$-glucosaminidase and $\alpha$-mannosidase. Activities of the following enzymes were not detected: trypsin, chymotrypsin, $\beta$-glucuronidase, $\alpha$-fucosidase, lysine decarboxylase, ornithine decarboxylase, urease, tryptophan deaminase and arginine dihydrolase.

Strain Ben $106^{\mathrm{T}}$ did not produce $\mathrm{H}_{2} \mathrm{~S}$ or indole. It was Voges-Proskauer-positive, produced acetoin, liquefied gelatin and reduced nitrate to nitrite. It was oxidasenegative, catalase-positive and a facultative anaerobe.

\section{Temperature and $\mathrm{pH}$ growth ranges}

Strain Ben $106^{\mathrm{T}}$ grew between 20 and $37^{\circ} \mathrm{C}$, with no growth occurring at 5,15 or $45^{\circ} \mathrm{C}$. The optimal growth temperature was $25^{\circ} \mathrm{C}$. It also grew at a pH range between 6.0 and 9.0 , with no growth occurring at $\mathrm{pH} 5.5$ and 9.3. The optimal $\mathrm{pH}$ for strain Ben $106^{\mathrm{T}}$ was $7 \cdot 5$.

\section{Phylogenetic characteristics}

Almost the complete 16S rRNA gene was sequenced (1410 bases) for strain Ben $106^{\mathrm{T}}$, corresponding to positions 9-1513 of Escherichia coli according to the nomenclature of Winker \& Woese (1991). Phylogeny based on 16S rRNA analysis indicated that the isolate was a member of Gram-positive Firmicutes, with a high $\mathrm{G}+\mathrm{C}$ content, in the domain Bacteria. The isolate formed a new branch within the family Propionibacteriaceae emend. Rainey, Ward-Rainey \& Stackebrandt (Stackebrandt et al., 1997) and was closest to members of the genera Propionibacterium as Propionibacterium propionicum (Charfreitag et al., 1988), Propioniferax (Pitcher \& Collins, 1991; Yokota et al., 1994), Friedmanniella (Schumann et al., 1997), Luteococcus (Tamura et al., 1994) and Microlunatus (Nak- 
Table 1. Comparative phenotypic characters of Propioniferax innocua, Propionibacterium propionicum, Luteococcus japonicus, Microlunatus phosphovorus, Friedmanniella antarctica and strain Ben $106^{\top}$

All isolates are Gram-positive, non-motile and non-spore-forming chemo-organotrophs. + , Positive test result; - , negative test result; v, variable test result;, $\pm 11-89 \%$ of the strains are positive; ND, not determined. Data obtained from (a) Pitcher \& Collins (1991) and Yokota et al. (1994), (b) Charfreitag et al. (1988), Schaal (1986) and Cummins \& Moss (1990), (c) Tamura et al. (1994), (d) Schumann et al. (1997), (e) Nakamura et al. (1995) and (f) this study.

\begin{tabular}{|c|c|c|c|c|c|c|}
\hline Phenotypic character & Propioniferax innocua & $\begin{array}{c}\text { Propionibacterium } \\
\text { propionicum }^{b}\end{array}$ & $\begin{array}{l}\text { Luteococcus } \\
\text { japonicus }\end{array}$ & $\begin{array}{c}\text { Friedmanniella } \\
\text { antarctica }^{4}\end{array}$ & $\begin{array}{l}\text { Microlunatus } \\
\text { phosphovorus }\end{array}$ & Strain Ben $106^{\mathrm{Tf}}$ \\
\hline $\mathrm{O}_{2}$ requirement & Facultative anaerobe & Facultative anaerobe & Facultative anaerobe & Aerobe & Aerobe & Facultative anaerobe \\
\hline Cell morphology & $\begin{array}{l}\text { Pleomorphic rods } \\
(0.5-1.2 \mu \mathrm{m})\end{array}$ & $\begin{array}{l}\text { Pleomorphic rods } \\
(0.2-0.8 \mu \mathrm{m})\end{array}$ & $\begin{array}{l}\text { Cocci }(0 \cdot 7-1 \cdot 0 \mu \mathrm{m}) \\
\text { arranged in pairs } \\
\text { and in tetrads }\end{array}$ & $\begin{array}{c}\text { Cocci }(0.5-2.2 \mu \mathrm{m}) \\
\text { arranged in packets }\end{array}$ & $\begin{array}{l}\text { Cocci }(0 \cdot 8-2 \cdot 0 \mu \mathrm{m}) \\
\text { arranged singly and } \\
\text { in pairs }\end{array}$ & $\begin{array}{l}\text { Cocci }(0 \cdot 5-1 \cdot 1 \mu \mathrm{m}) \\
\text { arranged in tetrads }\end{array}$ \\
\hline Habitat & $\begin{array}{c}\text { Human epidermal } \\
\text { surface }\end{array}$ & $\begin{array}{l}\text { Human oral cavity and } \\
\text { cervicovaginal secretion }\end{array}$ & Soil and water & Antarctic sandstone & $\begin{array}{l}\text { Sewage treatment plant } \\
\text { in Japan }\end{array}$ & $\begin{array}{l}\text { Sewage treatment plant } \\
\text { in Australia }\end{array}$ \\
\hline Optimum growth temperature $\left({ }^{\circ} \mathrm{C}\right)$ & 37 & $35-37$ & $26-28$ & 22 & $25-30$ & 25 \\
\hline Growth temperature range $\left({ }^{\circ} \mathrm{C}\right)$ & $10-40$ & $30-37$ & $12-38$ & $9-25$ & $5-35$ & $20-37$ \\
\hline Optimum growth $\mathrm{pH}$ & $7 \cdot 0$ & ND & ND & $6 \cdot 0-7 \cdot 2$ & 7.0 & $7 \cdot 5$ \\
\hline Growth pH range & ND & $\mathrm{ND}$ & ND & $5 \cdot 1-8 \cdot 7$ & $5 \cdot 0-9 \cdot 0$ & $5 \cdot 5-9 \cdot 3$ \\
\hline $\begin{array}{l}\text { Presence of metachromatic } \\
\text { granules* }\end{array}$ & $\begin{array}{l}+, \text { type of storage } \\
\text { granules unknown }\end{array}$ & ND & ND & $+^{\dagger}$, polyphosphate & + , polyphosphate & + , polyphosphate \\
\hline Oxidase & + & $\mathrm{ND}$ & + & - & + (weak) & - \\
\hline Catalase & + & - & + & $+\dagger$ & + & + \\
\hline Production of indole & - & - & - & - & + & - \\
\hline Major menaquinone & MK $-9\left(\mathrm{H}_{4}\right)$ & MK-9( $\left.\mathrm{H}_{4}\right)$ & MK-9 $\left(\mathrm{H}_{4}\right)$ & MK-9( $\left(\mathrm{H}_{4}\right)$ & MK-9(H) & $\begin{array}{c}\text { MK- } 9\left(\mathrm{H}_{4}\right) \text { and MK- } \\
7\left(\mathrm{H}_{4}\right)\end{array}$ \\
\hline Diamino acid/murein type & $\mathrm{LL}-\mathrm{A}_{2} \mathrm{pm} / \mathrm{A} 3-\gamma$ & $L L-A_{2} \mathrm{pm} / \mathrm{A} 3-\gamma^{\prime}$ & $\mathbf{L L}-\mathbf{A}_{2} \mathrm{pm} / \mathrm{A} 3-\gamma$ & $\mathrm{LL}-\mathrm{A}_{2} \mathrm{pm} / \mathrm{A} 3-\gamma^{\prime}$ & LL-A ${ }_{2} \mathrm{pm} / \mathrm{A} 3-\gamma^{\prime}$ & LL-A $\mathbf{A}_{2} \mathrm{pm} / \mathrm{A} 3-\gamma^{\prime}$ \\
\hline Polar lipid $\ddagger$ & PE, PG, PL, GL & ND & DPG, PG, PI, GL & PI, PG, DPG, PL & PI, PG, DPG, PL & PI, PG, DPG, PL \\
\hline Urease & + & - & - & + & $+\dagger$ & - \\
\hline Nitrate reduction & + & + & - & - & $+\dagger$ & + \\
\hline $\mathrm{G}+\mathrm{C}(\mathrm{mol} \%)$ & $59-63$ & $63-65$ & $66-68$ & 73 & 68 & 74 \\
\hline \multicolumn{7}{|l|}{ Carbohydrates utilized $\S$} \\
\hline Lactose & - & \pm & ND & - & $-\dagger$ & + \\
\hline Raffinose & - & + & + & - & $-\dagger$ & + \\
\hline Mannitol & - & + & + & - & $+\dagger$ & + \\
\hline Glycerol & + & \pm & + & - & $-\dagger$ & + \\
\hline Arabinose & - & - & $\begin{array}{l}+ \text { for } D \text {-arabinose } \\
\text { - for L-arabinose }\end{array}$ & $+($ weak $)$ & $+\dagger$ & + \\
\hline Inositol & - & \pm & + & - & $+\dagger$ & - \\
\hline Sucrose & + & + & + & - & -+ & + \\
\hline Fructose & + & + & + & - & -+ & + \\
\hline Glucose & + & + & + & - & $-\dagger$ & + \\
\hline Mannose & + & \pm & + & - & $-\dagger$ & + \\
\hline Galactose & $\mathrm{v}$ & \pm & + & - & $-\dagger$ & + \\
\hline Trehalose & + & \pm & + & - & $-\dagger$ & - \\
\hline Maltose & + & $\bar{t}$ & + & - & -+ & + \\
\hline Ribose & ND & \pm & + & + & $+\dagger$ & + \\
\hline Xylose & ND & - & - & + (weak) & $-\dagger$ & + \\
\hline Rhamnose & - & - & - & - & $-\dagger$ & - \\
\hline Adonitol & - & \pm & $\mathrm{ND}$ & - & $-\dagger$ & - \\
\hline
\end{tabular}

*Microlunatus, Friedmanniella and strain Ben 107 contain polyphosphate granules; the granule type for Propioniferax innocua has not been determined.

$\dagger$ Data obtained by using the Biolog and Microbact 24E systems (A. M. Maszenan and others, unpublished data).

$\ddagger$ DPG, diphosphatidylglycerol; GL, unknown glycolipid; PE, phosphatidylethanolamine; PG, phosphatidylglycerol; PI, phosphatidylinositol; PL, unknown phospholipid.

$\S$ Tested for Microlunatus phosphovorus and Ben $106^{\mathrm{T}}$ under aerobic conditions.

amura et al., 1991, 1995a). Bootstrap analysis demonstrates this relationship was robust with a high confidence value (Fig. 2).

\section{Chemotaxonomic characteristics}

The peptidoglycan of strain Ben $106^{\mathrm{T}}$ contained LLdiaminopimelic acid (LL- $\left.\mathrm{A}_{2} \mathrm{pm}\right)$ and its interpeptide bridge consisted of a single glycine residue. Position 1 of the peptide subunit was occupied by glycine, consistent with the cell wall being of the rare pepti- doglycan type A3- $\gamma^{\prime}$ described by Schleifer \& Seidl (1985). The major isoprenoid quinones were MK$9\left(\mathrm{H}_{4}\right)$ and MK-7 $\left(\mathrm{H}_{4}\right)$ with a peak area ratio of $50: 32$ (data summarized in Table 1). Its polar lipids consisted of phosphatidylinositol, phosphatidylglycerol, diphosphatidylglycerol and three unknown glycolipids, one of these unknown glycolipids being detected in high amounts. The fatty acid profile of Ben $106^{\mathrm{T}}$ was characterized by the predominance of anteiso-12methyltetradecanoic acid $\left(\mathrm{C}_{15: 0}\right)$ (Table 2) and its $\mathrm{G}+\mathrm{C}$ content was $74 \mathrm{~mol} \%$. 
Table 2. Percentage cellular fatty acid composition of Ben $106^{\top}$, Friedmanniella antarctica, Luteococcus japonicus, Microlunatus phosphovorus, Propioniferax innocua and Propionibacterium propionicum

The cellular fatty acids iso- (i) and anteiso- (ai) $\mathrm{C}_{15: 0}$ and $\mathrm{C}_{17: 0}$ predominate in Propionibacterium propionicum. $\mathrm{C}_{16: 0}$, hexadecanoic acid; $\mathrm{i}-\mathrm{C}_{15: 0}, 13$-methyltetradecanoic acid; ai- $\mathrm{C}_{15: 0}, 12$ methyltetradecanoic acid.

\begin{tabular}{|c|c|c|c|c|c|}
\hline Fatty acid & Ben $106^{\mathrm{T}}$ & $\begin{array}{c}\text { Friedmanniella } \\
\text { antarctica }^{*}\end{array}$ & $\begin{array}{l}\text { Luteococcus } \\
\text { japonicus* }\end{array}$ & $\begin{array}{c}\text { Microlunatus } \\
\text { phosphovorus* }\end{array}$ & $\begin{array}{c}\text { Propioniferax } \\
\text { innocua }\end{array}$ \\
\hline $\mathrm{C}_{13: 1}$ & $2 \cdot 0$ & - & - & - & - \\
\hline $\mathrm{i}-\mathrm{C}_{14: 0}$ & $4 \cdot 1$ & $2 \cdot 3$ & - & $4 \cdot 0$ & $3 \cdot 2$ \\
\hline$C_{14: 0}$ & $3 \cdot 1$ & - & 1.0 & $2 \cdot 9$ & - \\
\hline $\mathrm{C}_{14: 1}$ & $2 \cdot 0$ & - & - & - & - \\
\hline $\mathrm{i}-\mathrm{C}_{15 ; 0}$ & $5 \cdot 0$ & $23 \cdot 2$ & - & $22 \cdot 4$ & $20 \cdot 9$ \\
\hline $\mathrm{i}-\mathrm{C}_{15: 1}$ & 3.6 & - & - & - & - \\
\hline ai- $C_{15: 0}$ & $47 \cdot 0$ & $54 \cdot 5$ & - & $43 \cdot 5$ & $46 \cdot 5$ \\
\hline$C_{15: 0}$ & - & - & $5 \cdot 6$ & - & - \\
\hline$C_{15: 0}^{10.0}$ & 6.2 & - & 11.7 & - & - \\
\hline $\mathrm{i}-\mathrm{C}_{16: 0}$ & $4 \cdot 7$ & $2 \cdot 4$ & - & $6 \cdot 2$ & $7 \cdot 3$ \\
\hline$i-C_{16: 1}^{16: 0}$ & $3 \cdot 9$ & $2 \cdot 9$ & - & - & $4 \cdot 5$ \\
\hline$C_{16: 0}$ & $5 \cdot 7$ & - & $4 \cdot 8$ & $7 \cdot 5$ & - \\
\hline$C_{16: 1}^{10.0}$ & 3.9 & - & $20 \cdot 5$ & - & - \\
\hline $\mathrm{i}-\mathrm{C}_{17 \cdot 0}$ & - & - & - & - & $2 \cdot 2$ \\
\hline ai- $C_{17: 0}^{170}$ & - & - & - & - & 6.7 \\
\hline $\mathrm{C}_{17: 0}$ & - & - & $2 \cdot 4$ & - & - \\
\hline $\mathrm{i}-\mathrm{C}_{17: 1}$ & $8 \cdot 1$ & - & - & - & 1.3 \\
\hline$a^{a}-C_{17: 1}$ & - & - & - & - & $2 \cdot 9$ \\
\hline$C_{17: 1}$ & - & - & $48 \cdot 1$ & - & - \\
\hline $\mathrm{C}_{18: 0}$ & - & - & - & $5 \cdot 6$ & - \\
\hline$C_{18: 1}^{1.0}$ & - & - & $3 \cdot 2$ & - & - \\
\hline$C_{18: 2}$ & - & 6.0 & - & - & - \\
\hline
\end{tabular}

* Data obtained from Schumann et al. (1997).

\section{DISCUSSION}

Cocci arranged in tetrads have been observed in biomass samples obtained from activated sludge systems from around the world (Carruci et al., 1994; Cech \& Hartman, 1993; Jenkins \& Tandoi, 1991; Liu et al., 1996; Maszenan et al., 1997; Matsuo, 1994; Matsuzawa \& Mino, 1991 ; Randall, 1994; Ubukata \& Takii, 1994; Ubukata, 1994) but only a few such organisms have been isolated in pure culture and characterized. This paper reports a previously undescribed Gram-positive, non-spore-forming, facultatively anaerobic chemo-organotroph with cells in tetrads.

Some Gram-positive bacteria with high $\mathrm{G}+\mathrm{C}$ contents have been suggested to play a major role in biological phosphorus removal (Bond et al., 1995; Nakamura et al., 1995b; Wagner et al., 1994) but the organisms responsible for phosphate removal in activated sludge have not yet been identified with any conviction (Jenkins \& Tandoi, 1991; Knight et al., 1995). Strain Ben $106^{\mathrm{T}}$ can accumulate PolyP aerobically, while no PHA was observed, but the factors affecting accumulation of these polymers are not known. Its physiology needs to be studied to determine whether its behaviour fits the empirical models of biological phosphorus removal in activated sludge (e.g. van Loosdrecht et al., 1997) or the model for phosphorus uptake by slowgrowing bacteria such as Microlunatus phosphovorus as proposed by Nakamura et al. (1995a).

DNA probes based on 16S rRNA sequence data should be used to identify this organism in situ so that its possible role in phosphate removal in activated sludge plants can be better assessed and its true similarity or otherwise to organisms sharing the same microscopic morphology (i.e. cocci in regular packets or in a tetrad) in activated sludge biomass resolved (Matsuo, 1994; Matsuzawa \& Mino, 1991). More isolates of this organism should be sought from activated sludge biomass so that their physiological and ecological diversity can be clarified to give a much clearer understanding of the possible role of Grampositive cocci in activated sludge systems and to clarify the taxonomic uncertainties discussed below.

Strain Ben $106^{\mathrm{T}}$ shares a similar coccoid morphology with Luteococcus japonicus (Tamura et al., 1994), Friedmanniella antarctica (Schumann et al., 1997) and 
Table 3. Comparison of the signature nucleotides of $16 \mathrm{~S}$ rDNA for the families Propionibacteriaceae and Nocardioidaceae (Stackebrandt et al., 1997) with Ben $106^{\top}$ and Friedmanniella antarctica

Differences in the signature nucleotides from the designated signatures described by Stackebrandt et al. (1997) to delineate families within the Actinobacteria and whose taxonomic implications are discussed in the text are indicated by bold type.

\begin{tabular}{|ccccc|}
\hline Position* $^{*}$ & Propionibacteriaceae & Nocardioidaceae & ${\text { Ben } \text { 106 }^{\mathbf{T}}}^{\text {Friedmanniella antarctica }}$ \\
\hline $66-103$ & A-T & G-C & A-T & A-T \\
328 & T & C & T & T \\
$370-391$ & C-G & G-C & C-G & C-G \\
$407-435$ & C-G & A-T & C-G & C-G \\
$602-636$ & A-T & G-T & G-T & G-T \\
$658-748$ & A-T & T-A & A-T & G-A \\
686 & G & T & A & G \\
780 & A & G & A & A \\
787 & C & A & C & C \\
819 & G & T & G & G \\
$825-875$ & A-T & G-C & A-T & A-T \\
$1409-1491$ & A-T & C-G & A-T & A-T \\
\hline
\end{tabular}

${ }^{*} E$. coli numbering.

Microlunatus phosphovorus (Nakamura et al., 1991, 1995a), but not the characteristic hemispherical, lipped, cells of the latter (Nakamura et al., 1995a) nor the pleomorphic rod morphology of Propionibacterium propionicum (Schaal, 1986; Charfreitag et al., 1988). On the criterion of $16 \mathrm{~S}$ rDNA sequence data, strain Ben $106^{\mathrm{T}}$ is related, but not especially closely, to Propionibacterium propionicum (Schaal, 1986; Charfreitag et al., 1988), Propioniferax innocua (Pitcher \& Collins, 1991; Yokota et al., 1994), Luteococcus japonicus (Tamura et al., 1994), Friedmanniella antarctica (Schumann et al., 1997) and Microlunatus phosphovorus (Nakamura et al., 1991, 1995a) (Fig. 2), all of which belong to the family Propionibacteriaceae (Stackebrandt et al., 1997).

Strain Ben $106^{\mathrm{T}}$ does not contain the cellular fatty acid 10 -methyl- $\mathrm{C}_{18: 0}$ or phosphatidylethanolamine, which supports the view that it does not belong to the genus Aeromicrobium (Miller et al., 1991; Tamura \& Yokota, 1994). Unsaturated fatty acids with $13,14,15$ and 16 carbon atoms are found in Ben $106^{\mathrm{T}}$ but not in Microlunatus phosphovorus or Propioniferax innocua (Table 2). Although Ben $106^{\mathrm{T}}$ and Microlunatus phosphovorus share a similar type of cell wall peptidoglycan, the presence of 14-methylpentadecenoic acid (iso- $\mathrm{C}_{16: 1}$ ) and 15-methylhexadecenoic acid (iso- $\mathrm{C}_{17: 1}$ ) and the absence of octadecanoic acid $\left(\mathrm{C}_{18: 0}\right)$ in Ben $106^{\mathrm{T}}$ would also support the view that it does not belong to the genus Microlunatus (Table 2). The absence of MK-8 $\left(\mathrm{H}_{4}\right)$ in Ben $106^{\mathrm{T}}$ clearly suggests that it does not belong to the genus Nocardioides (Collins et al., 1994; Tamura \& Yokota, 1994). In addition to the predominant menaquinone $\mathrm{MK}-\left(9 \mathrm{H}_{4}\right)$, strain Ben $106^{\mathrm{T}}$ contains an additional menaquinone, $\mathrm{MK}-7\left(\mathrm{H}_{4}\right)$, in substantial amounts, thus differing from all other genera in the currently recognized family Propionibacteriaceae. It also differs from Propioniferax innocua as its cell wall peptidoglycan contains glycine at position 1 of the peptide subunit, while Propioniferax innocua has L-alanine at that position (Schumann et al., 1997), although Yokota et al. (1994) erroneously reported glycine at the same position. These chemotaxonomic data, summarized in Table 1, strongly support the other phenotypic data and phylogenetic data that Ben $106^{\mathrm{T}}$ does not belong to any of these genera.

Based on 16S rDNA sequence data, Ben $106^{\mathrm{T}}$ is only 95.9\% similar to its closest relative Propionibacterium propionicum. A comparison of its 16S rDNA signature nucleotides with members of the family Propionibacteriaceae reveals many similarities but also several differences to those suggested by Stackebrandt et al. (1997) for circumscribing members of this family. These are summarized in Tables 3 and 4 . For example, at positions 602-636 and 686 (E. coli numbering system) Ben $106^{T}$ has the nucleotide signature $G-T$ instead of A-T and A instead of G (Table 3). Similarly, Ben $106^{\mathrm{T}}$ differs markedly from members of the family Nocardioidaceae. In addition, Ben $106^{\mathrm{T}}$ also has a different nucleotide signature at position 671-735 to that considered diagnostic for the suborder Propionibacterineae (i.e. A-T), possessing $\mathrm{T}-\mathrm{A}$ instead (Table 4).

It is possible that the description of the family Propionibacteriaceae within the suborder Propionibacterineae (Stackebrandt et al., 1997) needs to be amended to include Ben $106^{\mathrm{T}}$ and Friedmanniella antarctica (Schumann et al., 1997), which also differs 
Table 4. 16S rDNA signature nucleotides for Ben 106', Friedmanniella antarctica and members of the families Propionibacteriaceae and Nocardioidaceae within the suborder Propionibacterineae

Differences in the signature nucleotides from the designated signatures described by Stackebrandt et al. (1997) to delineate families within the Actinobacteria and whose taxonomic implications are discussed in the text are indicated by bold type.

\begin{tabular}{|cccccc|}
\hline Position* & Propionibacterineae & Propionibacteriaceae & Nocardioidaceae & $\begin{array}{c}\text { Ben 106 }^{\mathbf{T}} \\
\text { antarctica }\end{array}$ & Friedmanniella $^{*}$ \\
\hline $127-234$ & A-T & A-T & A-T & A-T & A-T \\
$603-635$ & A-T & A-T & A-T & A-T & A-T \\
$657-735$ & G-C & G-C & G-C & G-C & G-C \\
$671-735$ & A-T & A-T & A-T & T-A & A-T \\
$986-1219$ & T-A & T-A & T-A & T-A & T-A \\
$987-1218$ & G-C & G-C & G-C & G-C & G-C \\
$990-1215$ & T-G & T-G & T-G & T-G \\
$1059-1198$ & C-G & C-G & C-G & C-G \\
\hline
\end{tabular}

* E. coli numbering.

in some of its signature nucleotides (see Tables 3 and 4). It is possible that Ben $106^{\mathrm{T}}$ and Friedmanniella may represent members of a new family within an amended suborder Propionibacterineae, but more isolates of these need to be characterized before this can resolved. Therefore, in spite of different signature nucleotides at some positions, strain Ben $106^{\mathrm{T}}$ is best placed currently in the family Propionibacteriaceae emend. Rainey, Ward-Rainey \& Stackebrandt 1997 (Stackebrandt et al., 1997) because of similarities in most of its phenotypic characteristics to members of this family.

However, our phylogenetic and chemotaxonomic data reveal that strain Ben $106^{\mathrm{T}}$ cannot be affiliated to any established genus within the family Propionibacteriaceae. Hence we propose that strain Ben $106^{\mathrm{T}}$ should be assigned to a new genus, Tessaracoccus gen. nov., with Tessaracoccus bendigoensis sp. nov. as type species.

\section{Description of Tessaracoccus gen. nov.}

Tessaracoccus (Tes.sa.ra'coc.cus. Gr. adj. tessara four; Gr. n. coccus grain; L. n. Tessaracoccus four round cells).

Gram-positive, non-spore-forming, facultatively anaerobic cocci, $0.5-1.1 \mu \mathrm{m}$ in diameter and arranged in regular tetrads. Cells are non-motile and accumulate polyphosphate. The organism is oxidase-negative, capable of reducing nitrate and catalase-positive. The cell wall contains $L \mathrm{~L}-\mathrm{A}_{2} \mathrm{pm}$ with a rare peptidoglycan type, A3- $\gamma^{\prime}$. The major menaquinones are MK-9( $\left.\mathrm{H}_{4}\right)$ and $\mathrm{MK}-7\left(\mathrm{H}_{4}\right)$. The main fatty acid is anteiso-12methyltetradecanoic acid and polar lipids are phosphatidylinositol, phosphatidylglycerol, diphosphatidylglycerol and three unknown glycolipids. The phylogenetic position of the genus is in the high-G $+\mathrm{C}$ content branch of Firmicutes with Microlunatus phosphovorus, Friedmanniella antarctica, Propioniferax innocua and Propionibacterium propionicum as its closest relatives. The $\mathrm{G}+\mathrm{C}$ content is $74 \mathrm{~mol} \%$. Type species is Tessaracoccus bendigoensis.

\section{Description of Tessaracoccus bendigoensis sp. nov.}

Tessaracoccus bendigoensis (ben.di.go'en. sis. M. L. adj. bendigoensis referring to Bendigo, the place of origin of the isolate).

The following sugars and sugar derivatives are utilized: L-arabinose, D-ribose, D-fructose, D-galactose, gentiobiose, $\alpha$-D-glucose, $\alpha$-D-lactose, lactulose, maltose, Dmannose, D-melibiose, sucrose, turanose, D-raffinose, D-xylose, stachyose, palatinose, maltotriose, methyl $\alpha$-D-glucoside, methyl $\alpha$-D-galactoside, methyl $\beta$-Dgalactoside, $\alpha$-cyclodextrin, $\beta$-cyclodextrin, dextrin, amygladin, arbutin, glycogen, D-mannitol, methylpyruvate, glucose 1-phosphate, glucose 6-phosphate, DL-glycerol phosphate, UMP, glycerol, uridine and salicin, but not cellobiose, L-fucose, D-psicose, Lrhamnose, D-trehalose, D-melezitose, 3-methylglucose, methyl $\beta$-D-glucoside, mannan, adonitol, D-arabitol, i-erythritol, $m$-inositol, D-sorbitol, xylitol, monomethylsuccinate, D-galacturonic acid lactone, 2aminoethanol, 2,3-butanediol, inulin, fructose 6-phosphate, methylsuccinate, adenosine, 2'-deoxyadenosine, AMP, TMP, D-tagatose, methyl $\alpha$-D-mannoside or sedoheptulosan. The following organic acids, amino acids and their derivatives are utilized: acetic acid, D-gluconic acid, $\alpha$-hydroxybutyric acid, DL-lactic acid, L-lactic acid and pyruvic acid, but not $c i s$-aconitic acid, citric acid, formic acid, D-galacturonic acid lactone, D-galacturonic acid, D-glucosaminic acid, Dglucuronic acid, $\beta$-hydroxybutyric acid, $\gamma$-hydroxybutyric acid, $p$-hydroxyphenylacetic acid, itaconic acid, $\alpha$-ketobutyric acid, $\alpha$-ketoglutaric acid, $\alpha$-ketovaleric acid, malonic acid, propionic acid, quinic acid, D-saccharic acid, sebacic acid, succinic acid, bromosuccinic acid, succinamic acid, L-aspartic acid, Lglutamic acid, glycyl-L-aspartic acid, glycyl-L-glutamic 
acid, L-pyroglutamic acid, $\gamma$-aminobutyric acid, uroconic acid, D-lactic acid methyl ester, D-malic acid, Lmalic acid and $N$-acetyl-L-glutamic acid. The following substrates are metabolized: $N$-acetyl-D-glucosamine and uridine. The following substrates are not metabolized: Tween 40, Tween $80, N$-acetyl-D-galactosamine, $\quad N$-acetylmannosamine, glucuronamide, alaninamide, lactamide, D-alanine, L-alanine, Lalanylglycine, L-asparagine, L-histidine, L-leucine, L-ornithine, L-phenylalanine, L-proline, D-serine, Lserine, L-threonine, DL-carnitine, inosine, thymidine, phenylethylamine, putrescine and hydroxy-L-proline. It possesses the following enzymes: alkaline phosphatase, esterase, esterase lipase, lipase, leucine arylamidase, valine arylamidase, cystine arylamidase, acid phosphatase, naphthol-AS-BI-phosphohydrolase, $\alpha$-galactosidase, $\beta$-galactosidase, $\alpha$-glucosidase, $\beta$ glucosidase, $N$-acetyl- $\beta$-glucosaminidase and $\alpha$-mannosidase. Cells lack trypsin, chymotrypsin, $\beta$-glucuronidase, $\alpha$-fucosidase, lysine decarboxylase, ornithine decarboxylase, urease, tryptophan deaminase and arginine dihydrolase. It does not produce $\mathrm{H}_{2} \mathrm{~S}$ and indole. It is Voges-Proskauer-positive and can produce acetoin. It can liquefy gelatin. The natural habitat is activated sludge. The temperature range for growth is between 20 and $37^{\circ} \mathrm{C} ; \mathrm{pH}$ range for growth is between 6.0 and $9 \cdot 0$. Optimal growth is at $25^{\circ} \mathrm{C}$ and at $\mathrm{pH} 7 \cdot 5$. The type strain, Ben $106^{\mathrm{T}}$, has been deposited in the Australian Collection of Microorganisms as strain ACM $5119^{\mathrm{T}}$.

\section{ACKNOWLEDGEMENTS}

We wish to thank Mr K. C. Lindrea for providing the samples of biomass and the plant operational parameters from the pilot plant. The financial assistance in part to B. K.C.P. from the Australian Research Council is gratefully acknowledged. This work was supported by a La Trobe University Central Grant and A.M.M. was funded by a LUPRS and OPRS scholarship. Mr John Penwill, La Trobe University, Bendigo, and Professor $\mathrm{H}$. Trüper are thanked for their assistance in naming the organism.

\section{REFERENCES}

Bond, P. L., Hugenholtz, P., Kellar, J. \& Blackall, L. L. (1995). Bacterial community structures of phosphate-removing and non-phosphate-removing activated sludges from sequencing batch reactors. Appl Environ Microbiol 61, 1910-1916.

Carucci, A., Majone, M., Ramadori, R. \& Rossetti, S. (1994). Dynamics of phosphorus and organic substrates in anaerobic and aerobic phases of a sequencing batch reactor. Water Sci Technol 30, 237-246.

Cech, J. S. \& Hartman, P. (1993). Competition between polyphosphate and polysaccharide accumulating bacteria in enhanced biological phosphate removal systems. Water Res 27, 1219-1225.

Charfreitag, O., Collins, M. D. \& Stackebrandt, E. (1988). Reclassification of Arachnia propionica as Propionibacterium propionicus comb. nov. Int J Syst Bacteriol 38, 354-357.

Collins, M. D., Cockcroft, S. \& Wallbanks, S. (1994). Phylogenetic analysis of a new LL-diaminopimelic acid-containing cory- neform bacterium from herbage, Nocardioides plantarum $\mathrm{sp}$. nov. Int J Syst Bacteriol 44, 523-526.

Cummins, C. S. \& Moss, C. W. (1990). Fatty acid composition of Propionibacterium propionicus (Arachnia propionica). Int J Syst Bacteriol 40, 307-308.

Felsenstein, J. (1993). PHYLIP (Phylogeny Inference Package) version 3.5.1. Seattle: Department of Genetics, University of Washington.

Jenkins, D. \& Tandoi, v. (1991). The applied microbiology of enhanced biological phosphate removal-accomplishments and needs. Water Res 25, 1471-1478.

Jukes, T. H. \& Cantor, C. R. (1969). Evolution of protein molecules. In Mammalian Protein Metabolism, vol. 3, pp. 21-132. Edited by H. N. Munro. New York: Academic Press.

Kataoka, N., Tokiwa, T., Tanaka, Y., Takeda, K. \& Suzuki, T. (1996). Enrichment culture and isolation of slow growing bacteria. Appl Microbiol Biotechnol 45, 771-777.

Knight, G. C., Seviour, E. M., Seviour, R. J., Soddell, J. A., Lindrea, K. C., Strachan, W., DeGrey, B. \& Bayly, R. C. (1995). Development of the microbial community of a full scale nutrient removal activated sludge plant during start up. Water Res 29, 2085-2093.

Liu, W.-T., Mino, T., Nakamura, K. \& Matsuo, T. (1996). Glycogen accumulating populations and its anaerobic substrates uptake in anaerobic-aerobic activated sludge without biological phosphorus removal. Water Res 30, 75-82.

van Loosdrecht, M. C. M., Hooijmans, C. M., Brdjanovic, D. \& Heijnen, J. J. (1997). Biological phosphate removal processes. Appl Microbiol Biotechnol 48, 289-296.

Maidak, B. L., Olsen, G. J., Larsen, N., Overbeek, R., McCaughey, M. J. \& Woese, C. R. (1996). The Ribosomal Database Project (RDP). Nucleic Acids Res 24, 82-85.

Maszenan, A. M., Seviour, R. J., Patel, B. K. C., Rees, G. N. \& McDougall, B. M. (1997). Amaricoccus gen. nov., a gramnegative coccus occurring in regular packages or tetrads isolated from activated sludge biomass. Description of four species belonging to the genus. Int J Syst Bacteriol 47, 727-734.

Matsuo, Y. (1994). Effect of the anaerobic solid retention time on enhanced biological phosphorus removal. Water Sci Technol 30, 193-202.

Matsuzawa, Y. \& Mino, T. (1991). Role of glycogen as an intracellular carbon reserve of activated sludge in the competitive growth of filamentous and non filamentous bacteria. Water Sci Technol 23, 899-905.

Miller, E. R., Woese, C. R. \& Brenner, S. (1991). Description of the erythromycin-producing bacterium Arthrobacter sp. strain NRRL B-3381 as Aeromicrobium erythreum gen. nov., sp. nov. Int $J$ Syst Bacteriol 41, 363-368.

Nakamura, K., Masuda, K. \& Mikami, E. (1991). Isolation of a new type of polyphosphate accumulating bacterium and its phosphate removal characteristics. J Ferment Technol 71, 258-263.

Nakamura, K., Hiraishi, A., Yoshimi, Y., Kawaharasaki, M., Masuda, K. \& Kamagata, Y. (1995a). Microlunatus phosphovorus gen. nov., sp. nov., a new gram-positive polyphosphateaccumulating bacterium isolated from activated sludge. Int $J$ Syst Bacteriol 45, 17-22.

Nakamura, K., Ishikawa, S. \& Kawaharasaki, M. (1995b). Phosphate uptake and release activity in immobilized polyphosphate accumulating bacterium Microlunatus phosphovorus strain NM1. J Ferment Technol 80, 377-382.

Owen, R. J. \& Lapage, S. P. (1976). The thermal denaturation of partly purified bacterial deoxyribonucleic acid and its taxonomic applications. J Appl Bacteriol 41, 335-340. 
Painter, H. A. (1983). Metabolism and physiology of aerobic bacteria and fungi. In Ecological Aspects of Used-Water Treatment: Vol.2, Biological Activities and Treatment Processes, pp. 11-25. Edited by C. R. Curds \& H. A. Hawks. London: Academic Press.

Patel, B. K. C., Andrews, K. T. , Ollivier, B., Mah, R. A. \& Garcia, J. L. (1995). Re-evaluating the classification of Halobacteroides and Haloanaerobacter species based on sequence comparisons of the 16S ribosomal RNA gene. FEMS Microbiol Lett 134, $115-119$.

Pitcher, D. G. \& Collins, M. D. (1991). Phylogenetic analysis of some LL-diaminopimelic acid-containing coryneform bacteria from human skin: description of Propionibacterium innocuum sp. nov. FEMS Microbiol Lett 84, 295-300.

Porteous, L. A., Armstrong, J. L., Seidler, R. J. \& Watrud, L. S. (1994). An effective method to extract DNA from environment samples for polymerase chain reaction amplification and DNA fingerprint analysis. Curr Microbiol 29, 301-307.

Randall, A. A. (1994). The effect of substrate chemistry on enhanced biological phosphorus removal, intracellular phosphate form and location and the resulting population structure of sequencing batch reactors receiving synthetic wastewater. $\mathrm{PhD}$ thesis, Auburn University, AL, USA.

Rees, G. N., Vasiliadis, G., May, J.W. \& Bayly, R. C. (1992). Differentiation of polyphosphate and poly- $\beta$-hydroxybutyrate granules in an Acinetobacter sp. isolated from activated sludge. FEMS Microbiol Lett 94, 171-174.

Schaal, K. P. (1986). Genus Arachnia Pine and Georg 1969, 269 AL In Bergey's Manual of Systematic Bacteriology, vol. 2, pp. 1332-1342. Edited by P. H. A. Sneath, N. S. Mair, M. E. Sharpe \& J. G. Holt. Baltimore: Williams \& Wilkins.

Schleifer, K. H. \& Kandler, O. (1972). Peptidoglycan types of bacterial cell walls and their taxonomic implications. Bacteriol Rev 36, 406-477.

Schleifer, K. H. \& Seidl, P. H. (1985). Chemical composition and structure of murein. In Chemical Methods in Bacterial Systematics, pp. 201-219. Edited by M. Goodfellow \& D. E. Minnikin. London: Academic Press.

Schumann, P., Prauser, H., Rainey, F. A., Stackebrandt, E. \& Hirsch, P. (1997). Friedmanniella antarctica gen. nov., sp. nov., an LL-diaminopimelic acid-containing actinomycete from Antarctic sandstone. Int J Syst Bacteriol 47, 278-283.

Skerman, V. B. D. (1968). A new type of micromanipulator and microforge. J Gen Microbiol 54, 287-297.
Stackebrandt, E., Rainey, F. A. \& Ward-Rainey, N. L. (1997). Proposal for a new hierarchic classification system, Actinobacteria classis nov. Int J Syst Bacteriol 47, 479-491.

Takii, S. (1977a). Accumulation of reserve polysaccharide in activated sludge treating carbohydrate wastes. Water Res 11, 79-83.

Takii, S. (1977b). Bacterial characteristic of activated sludges treating carbohydrate waste. Water Res 11, 85-89.

Tamura, T. \& Yokota, A. (1994). Transfer of Nocardioides fastidiosa Collins and Stackebrandt 1989 to the genus Aeromicrobium as Aeromicrobium fastidiosum comb. nov. Int $J$ Syst Bacteriol 44, 608-611.

Tamura, T., Takeuchi, M. \& Yokota, A. (1994). Luteococcus japonicus gen. nov., sp. nov., a new gram-positive coccus with LL-diaminopimelic acid in the cell wall. Int J Syst Bacteriol 44, 348-356.

Ubukata, Y. (1994). Some physiological characteristics of a phosphate removing bacterium isolated from anaerobic/ aerobic activated sludge. Water Sci Technol 30, 229-235.

Ubukata, Y. \& Takii, S. (1994). Induction method of excess phosphate accumulation for phosphate removing bacteria isolated from anaerobic/aerobic activated sludge. Water Sci Technol 30, 221-227.

Wagner, M., Erhart, R., Manz, W., Amann, R., Lemmer, H., Wedi, D. \& Schleifer, K.-H. (1994). Development of rRNA-targeted oligonucleotide probe specific for the genus Acinetobacter and its application for in situ monitoring in activated sludge. Appl Environ Microbiol 60, 792-800.

Wanner, J. (1994). Activated Sludge Bulking and Foaming Control. Lancaster: Technomic Publishing Company.

Williams, T. M. \& Unz, R. F. (1985). Isolation and characterization of filamentous bacteria present in bulking activated sludge. Appl Microbiol Biotechnol 22, 273-282.

Winker, S. \& Woese, C. R. (1991). A definition of the domain Archaea, Bacteria and Eucarya in terms of small ribosomal RNA characteristics. Syst Appl Microbiol 14, 305-310.

Yokota, A., Tamura, T., Takeuchi, M., Weiss, N. \& Stackebrandt, E. (1994). Transfer of Propionibacterium innocuum Pitcher and Collins 1991 to Propioniferax gen. nov. as Propioniferax innocua comb. nov. Int J Syst Bacteriol 44, 579-582.

Yoshimi, Y., Hiraishi, A. \& Nakamura, K. (1996). Isolation and characterization of Microsphaera multipartita gen. nov., sp. nov., a polysaccharide-accumulating gram-positive bacterium from activated sludge. Int $J$ Syst Bacteriol 46, 519-525. 